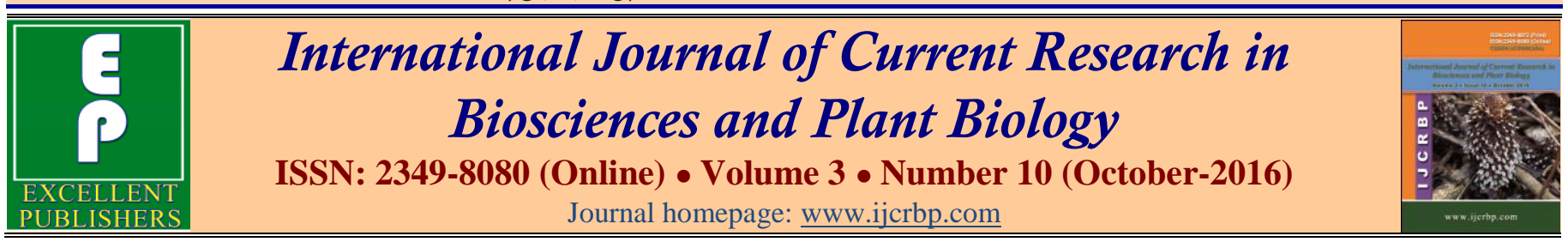

Original Research Article

doi: http://dx.doi.org/10.20546/ijcrbp.2016.310.019

\title{
Molecular Cloning and Sequence Analysis of a Phosphomevalonate Kinase Gene (CnPMK) from Chamaemelum nobile
}

\author{
Jiaping Yan, Xiangxiang Meng, Feng $X u^{*}$ and Jie Chang* \\ College of Horticulture and Gardening, Yangtze University, Jingzhou 434025, China \\ *Corresponding authors.
}

\begin{abstract}
A bstract
Roman chamomile (Chamaemelum nobile L.) is renowned for its production of sesquiterpenoids, and specifically sesquiterpene lactones. Phosphomevalonate Kinase gene $(P M K)$ is one of the core enzymes in the biosynthesis pathway of sesquiterpenoids, which catalyzes the formation ofmevalonate-5-diphosphate. To isolate and identify the key genes involved in the sesquiterpenoids biosynthesis of the $C$. nobile, a $P M K$ gene designated as CnPMK (GenBank Accession No. KX894316) was cloned from C. nobile. The full-length cDNA of CnPMK was 1942bp and contained a 1482bp open reading frame (ORF), which encoding a 493 amino-acid protein. The molecular weight and $\mathrm{pI}$ of the CnPMK protein are $53.8 \mathrm{KDa}$ and 5.55 , respectively. The encoding amino-acid sequence of the CnGAS showed high similarity to other plant PMK proteins. Phylogenetic tree analysis revealed that CnPMK clustered with the PMK of Asteraceae in the dicotyledons clade. The $C n P M K$ gene was isolated from $C$. nobile, which will lay a foundation for further study of sesquiterpenoid biosynthesis pathway in C. nobile.
\end{abstract}

Article Info

\section{Introduction}

Roman chamomile (Chamaemelum nobile L.) is a perennial herbaceous plant of the Asteraceae family found in wild and cultivated in western Europe, North America and northern Africa and Asia (Ma et al., 2007). As a traditional medical plant, Roman chamomile is considered to be an antiseptic, antibiotic, disinfectant, bactericidal, fungicidal and vermifuge (Guimarães et al., 2013). This herb is often used against nausea, vomiting, indigestion, and loss of appetite (Srivastava et al., 2010). The biological activity of chamomile is mainly due to the flavonoids apigenin, luteolin, quercetin, patuletin and terpenoids chamazulene, sesquiterpene lactones, bisabolol and its oxides and azulenes (Hadaruga et al., 2009).

Terpenoids are synthesized by a variety of terpenoid synthases that constitutes a highly diverse gene family producing a wide range of cyclic and acyclic molecules consisting of isoprene (C5) residues. Over 60,000 terpenes and derivatives are found in nature (Cheng et al., 2007; Bohlmann and Keeling, 2008). Terpenoids are synthesized in plants through two independent pathways: the mevalonate (MVA) pathway in the cytoplasm and the 2Cmethyl-D-erythritol 4-phosphate (MEP) pathway in the plastids (Fig. 1, Vranová et al., 2013). During recent decades, there has been great progress in identification and functional characterization of terpenoid biosynthesis genes, enzymes and in metabolic engineering of terpenoid synthesis, and this has contributed greatly to improved understanding of basic mechanisms and variability of terpenoid biosynthesis (Chen et al., 2011; Rajabi Memari et al., 2013). In particular, proteomics data has previously shown that the fourth and fifth enzymes in the MVA 
pathway-mevalonate kinase (MK) and phosphormevalonate kinase (PMK), respectively - are expressed at relatively low levels and may be targets for increasing overall isoprenoid production (Redding-Johanson et al., 2011; Singh et al., 2012). Previous study has also shown that substrate inhibition and feedback inhibition of MK may be responsible for limiting flux through the pathway (Ma et al., 2011).

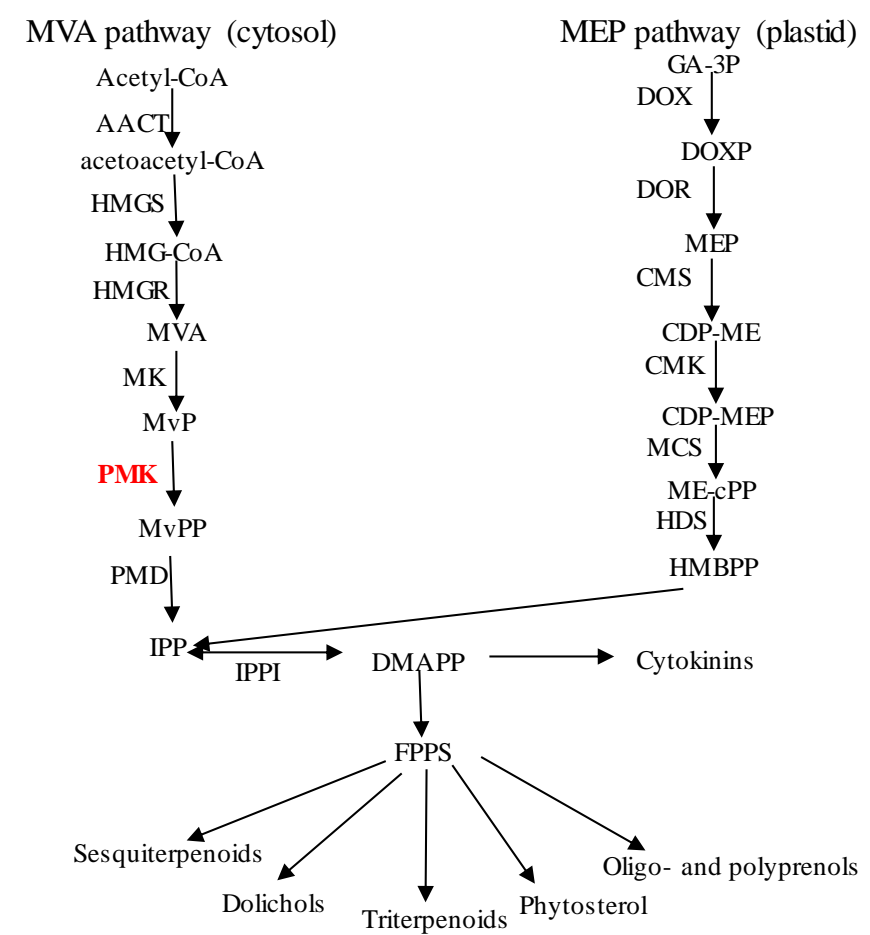

Fig. 1: Isoprenoid biosynthetic pathways in the plant cell.

As an important enzyme in the MVA pathway, PMK catalyzes the reaction of mevalonate-5-phosphate (MvP) and ATP to form mevalonate-5-disphosphate (MvPP) and ADP (Miziorko, 2011), which is further converted into isopentenyl diphosphate (IPP) by the mevalonate diphosphate decarboxylase (PMD). All the isoprenoids are derived from IPP precursors, and the IPP is generated by the pyrophosphorylation and decarboxylation of MVA; common precursors are used for the biosynthesis of terpenoids such as mono-, sesqui-, di-, and triterpenoids. Normally, sesquiterpene lactones are synthesized from MVA pathway. Given its importance in the terpenoids biosynthesis, some $P M K$ genes have been studied for its function in the MVA pathway. PMK is found in eukaryotes and some Eubacteria. The amino acid sequence for animal and low homology invertebrate PMK proteins are not orthologous to those for PMK in plants, fungi, and bacteria (Smit and Mushegian, 2000). Thus, the proteins that catalyze the enzymatic reaction differ widely, depending on their source. Although
Roman chamomile is an important medical plant, few studies focused on identifying the enzymes or genes involved in sesquiterpenoid biosynthesis. To investigate the whole biosynthesis pathway of terpenoids in Roman chamomile, each gene involved in this pathway should be identified and characterized. Here, we report the cloning of the full-length cDNA of the $P M K$ gene from Roman chamomile for the first time. We also analyzed the structure of the sequence, aiming to add the gene source for increasing the content of sesquiterpenoids in $C$. nobile through the genetic engineering.

\section{Materials and methods}

Erect Roman chamomile (C. nobile) plants were grown under controlled conditions in a growth chamber $(16 \mathrm{hrs}$ day length and day/night temperature of $23 / 18^{\circ} \mathrm{C}$, incident quantum flux density of $400 \mu \mathrm{mol} \mathrm{m} \mathrm{m}^{-2} \mathrm{~s}^{-1}$ ). Roman chamomile leaves were collected and immediately frozen in liquid nitrogen and stored at a $80^{\circ} \mathrm{C}$ for RNA extraction. MiniBEST Plant RNA Extraction kit, PrimeScript ${ }^{\mathrm{TM}} 1^{\text {st }}$ Strand cDNA Synthesis Kit, Agarose Gel DNA purification Kit Ver.4.0 and pMD18-T vector kit were purchased from TaKaRa Company (Dalian, China). Both the primers synthesis and DNA sequencing were performed by Shanghai Sangon Biotechnology Company, in China.

\section{Cloning the full-length cDNA of CnPMK}

Total RNA was isolated from frozen plant tissues using the TaKaRa MiniBEST Plant RNA Extraction kit. The first strand cDNA was synthesized according to the instruction of PrimeScript ${ }^{\mathrm{TM}} 1 \mathrm{st}$ Strand cDNA Synthesis Kit. Five ESTS, showed a high similarity level with the beginning and the end of queries, respectively. Primers CnPMK-up (5'-ATGTCTGTGGTTGCTTCGGCTC-3') and CnPMKdn (5'-TTACTTTTCCCGAGGATCACCACT-3') were designed on Group1_Unigene_BMK.5730 sequence to investigate the full length. CnPMK cDNA was amplified under the following conditions: $94^{\circ} \mathrm{C}$ for $4 \mathrm{~min}$; 30 cycles of amplification at $94^{\circ} \mathrm{C}$ for $30 \mathrm{~s}, 63^{\circ} \mathrm{C}$ for $30 \mathrm{~s}$, and $72^{\circ} \mathrm{C}$ for $90 \mathrm{~s}$; and a final extension at $72^{\circ} \mathrm{C}$ for $10 \mathrm{~min}$. The amplified products were analyzed by $1 \%$ gel electrophoresis and purified by Agarose Gel DNA purification Kit Ver.4.0. The purified products were ligated into the pMD18-T vector, and then cloned into the Escherichia coli strain DH5 $\alpha$ followed by sequencing.

\section{Bioinformatic analysis}

The obtained nucleotide sequence and deduced amino 
acid sequence were analyzed by the bioinformatics software on websites (http://www.ncbi.nlm.nih.gov/ BLAST/ and http://web. expasy.org/ protparam/). Plant PMK protein sequences were retrieved from NCBI GenBank. Multiple sequence alignments were performed using Vector NTI 11.5 program and phylogenetic analysis was conducted using MEGA 6.0 software. The neighbor-joining method was used to construct a guided tree, supported by boostrapping based on 1000 replicates.

\section{Results and discussion}

\section{Cloning and characterization of $C n P M K$}

The cDNA of CnPMK (GenBank Accession No. KX894316) was cloned by a pair of specific primers based on CnPMK unigene of transcriptome data. The PCR products were sequenced, and results showed that the cDNA sequence of the PCR products was 1942bp and contained a 1482bp open reading frame (ORF), which encoding a 493 amino-acid protein (Fig. 2).

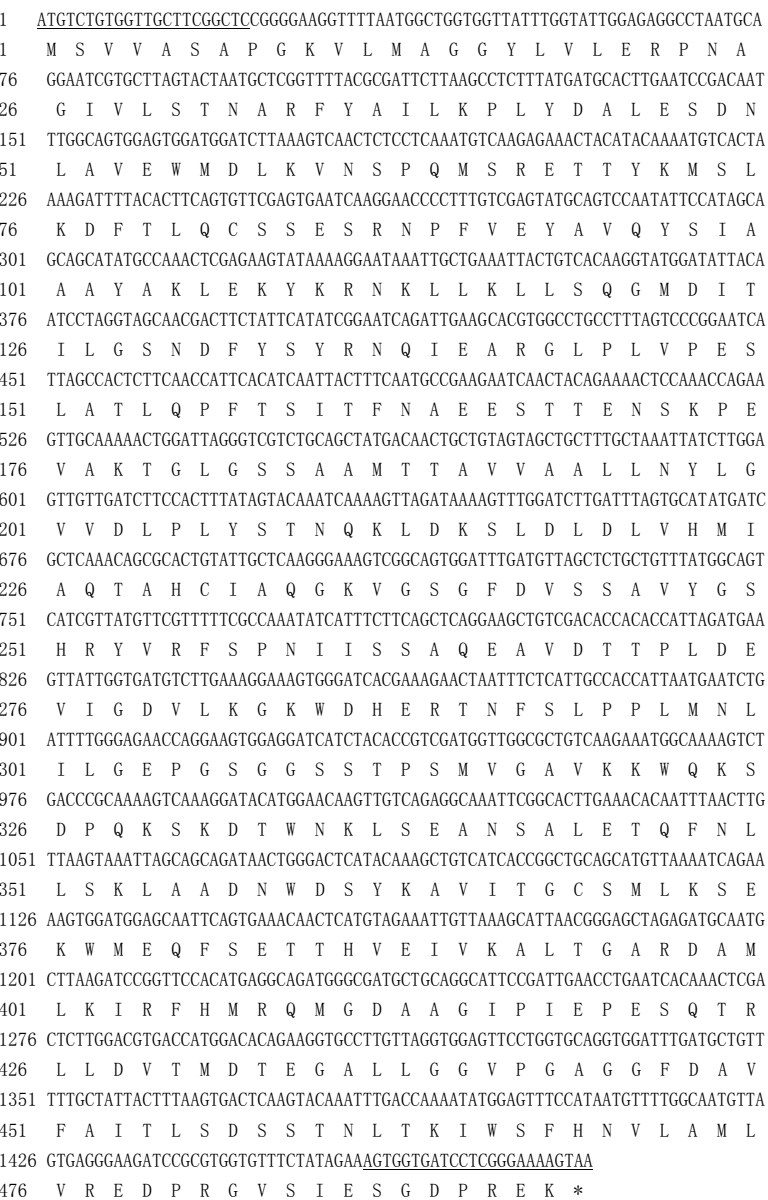

Fig. 2: Nucleotide sequence and deduced amino acid sequence of $C n P M K$. The primer sequences are underlined. Asterisks represent the termination codon.
The nucleotide sequence of $C n P M K$ had a high similarity with other $P M K$ genes. The nucleotide sequence of CnPMK was $85 \%, 76 \%, 76 \%, 76 \%, 75 \%$, and $74 \%$ identical to those of the $P M K$ genes from Taraxacum koksaghyz, Daucus carota subsp. sativus, Citrus sinensis, Citrus clementina, Ziziphus jujube, and Hevea brasiliensis (Table 1). Thus, the results indicated that the gene we cloned might be a member of the $P M K$ gene family.

Table 1. Nucleotide sequence of $C n P M K$ similarity to the $P M K$ genes from other plant species

\begin{tabular}{lll}
\hline Species & Accession No. & Homology \\
\hline Taraxacum kok-saghyz & KT899411.1 & $85 \%$ \\
Daucus carota subsp. sativus & XM_017374652.1 & $76 \%$ \\
Citrus sinensis & XM_006471412.2 & $76 \%$ \\
Citrus clementina & XM_006432616.1 & $76 \%$ \\
Ziziphus jujuba & XM_016012840.1 & $75 \%$ \\
Hevea brasiliensis & JN036535.1 & $74 \%$ \\
\hline
\end{tabular}

\section{Characterization of the deduced CnPMK protein}

The deduced CnPMK protein contained 493 amino acids. The theoretical molecular weight and isoelectric point (pI) of the deduced CnPMK protein were predicted to be $53.8 \mathrm{KDa}$ and 5.55 , respectively. Multiple sequence alignment of CnPMK amino acid sequences with PMKs from other species showed high sequence similarity (Fig. 3). The CnPMK protein showed 75\%, 76\%, 73\%, $73 \%, 71 \%, 73 \%, 73 \%$, and $71 \%$ identical to the counterparts of D. carota subsp. sativus, Panax notoginseng, Populus euphratica, Ricinus communis, Tripterygium wilfordii, Morus alba, Z. jujube, and Siraitia grosvenorii, respectively. The multiple alignments analysis revealed that CnPMK represents the plant and fungal forms of the ERG8 type of PMK (Pmev_kin_ERG8 type, eukaryotic branch). So far, two nonorthologous genes encoding PMK have been described, the Saccharomyces cerevisiae ERG8 gene and the human PMK gene. Houten and Waterham (2001) reported that orthologues of ERG8 are present in Eubacteria, fungi, and plants, while orthologues of human PMK are found only in animals, indicative of a nonorthologous gene displacement early in animal evolution.

\section{Molecular evolution analysis}

To investigate the evolutionary relationships in CnPMK and PMK proteins from other species, we selected the typical PMK proteins from the GenBank. We constructed a phylogenetic tree using the software MEGA 6.0 with neighbor-joining (NJ) method to analyze the molecular evolution of CnPMK on the basis of the three groups 
(Dicotyledoneae, Monocotyledoneae and bacteria) and ten families of Angiosperms. As shown in Fig. 4, CnPMK belonged to the Asteraceae in the branch of Dicotyledoneae; the CnPMK protein had a close relationship to TkPMK of T. kok-saghyz. Taken together, these results indicated that CnPMK shared common evolutionary originals with other PMK proteins based on conserved sequence structure and sequence characteristics.

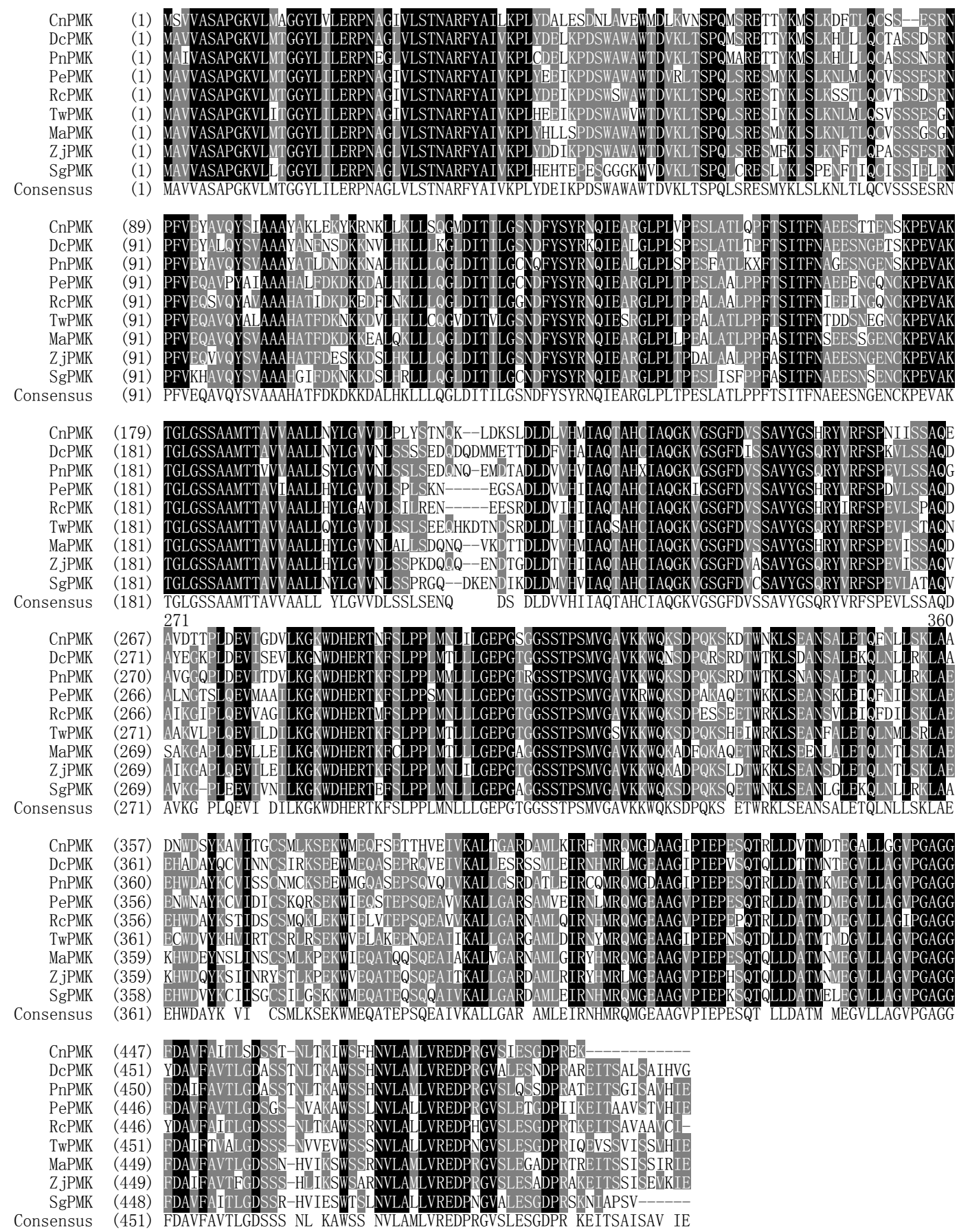

Fig. 3: Sequence multi-alignment of the deduced CnPMK protein with other PMK proteins. The species name and GenBank accession number are shown as following: CnPMK, Chamaemelum nobile; DcPMK, D. carota subsp. sativus (XP_017223228.1); PnPMK, P. notoginseng (AIK21784.1); PePMK, P. euphratica (XP_011028097.1); RcPMK, $R$. communis (XP_002520206.1); TwPMK, T. wilfordii (AMB15002.1); MaPMK, M. alba (ALD84323.1); ZjPMK, Z. jujube (XP_015868326.1); SgPMK, S. grosvenorii (AEM42973.1). Shaded in black are identical sequence. Shaded in gray are conservative sequence. 


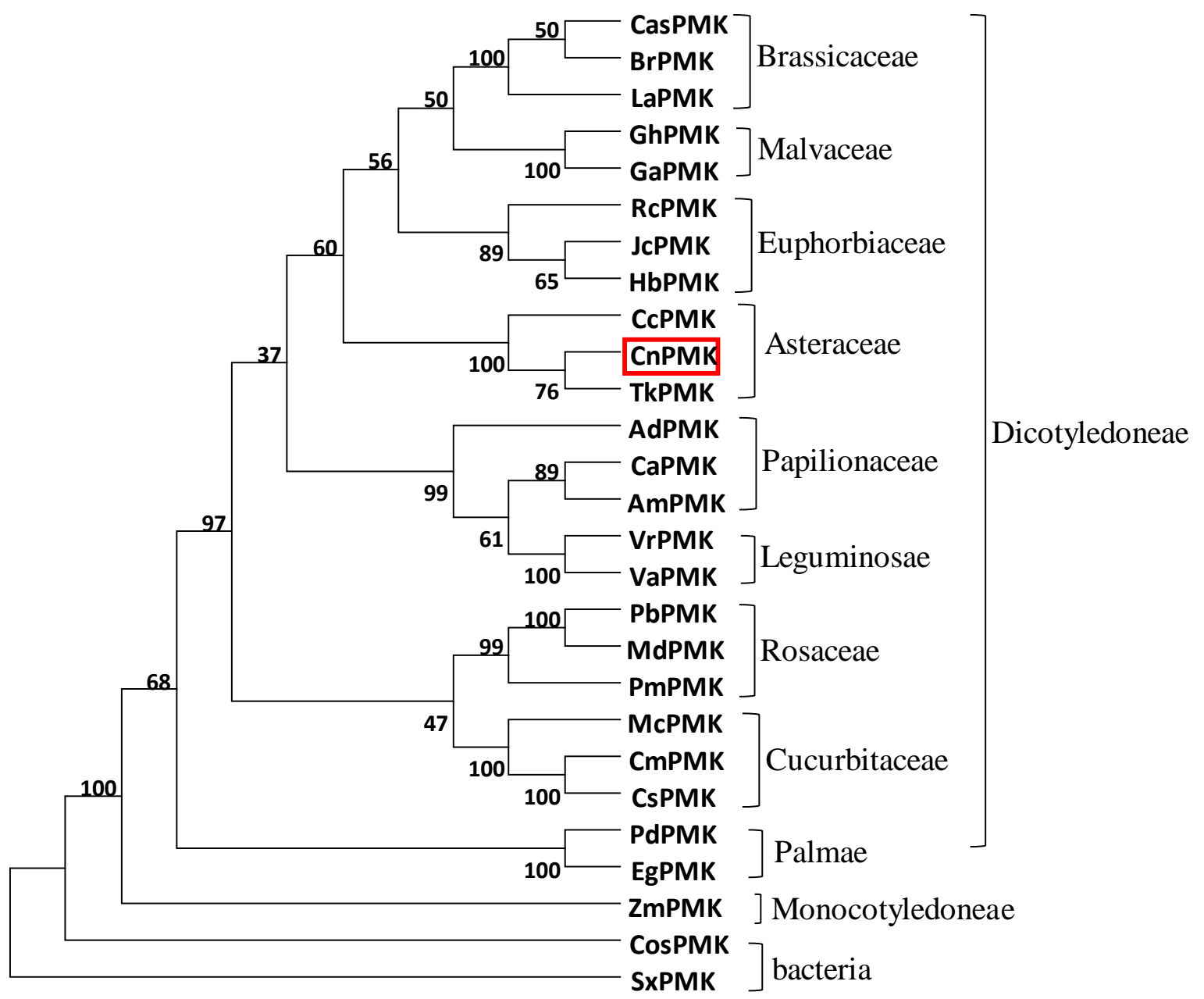

Fig. 4: The phylogenetic tree of phosphomevalonate kinase including CnPMK. Phylogenetic analysis of $C n P M K$ with other PMK proteins from other plants and bacteria. Bootstrap values are expressed in percentages and placed at the nodes in the tree. The GenBank accession No. of the PMK proteins and species are as following: CasPMK, Camelina sativa (XP_010478612.1); BrPMK, Brassica rapa (XP_009108002.1); LaPMK, Lepidium apetalum (ALJ53305.1); GhPMK, Gossypium hirsutum (XP_016736729.1); GaPMK, Gossypium arboretum (XP_017638066.1); JcPMK, Jatropha curcas (XP_012092013.1); HbPMK, H. brasiliensis (AFJ74328.1); CcPMK, Cynara cardunculus (KVH95774.1); TkPMK, Taraxacum kok-saghyz (AMB19700.1); AdPMK, Arachis duranensis (XP_015934336.1); CaPMK, Cicer arietinum (XP_004502634.1); AmPMK, Astragalus membranaceus (AID51440.1); VrPMK, Vigna radiate (XP_014519068.1); VaPMK, Vigna angularis (XP_017422173.1); PbPMK, Pyrus × bretschneideri (XP_009341974.1); MdPMK, Malus domestica (XP_008368652.2); PmPMK, Prunus mume (XP_016651369.1); McPMK, Momordica charantia (AKO82483.1); CmPMK, Cucumis melo (XP_008466028.1); CsPMK, Cucumis sativus (XP_004136210.1); PdPMK, Phoenix dactylifera (XP_008788523.1); EgPMK, Elaeis guineensis (XP_010920023.1); ZmPMK, Zea mays (NP_001149345.1); CosPMK, Colletotrichum sublineola (KDN67694.1); SxPMK, Staphylococcus xylosus (KTW24759.1).

\section{Conclusion}

In this study, a $P M K$ homologue, $C n P M K$ was successfully cloned and characterized for the first time from a Asteraceae plant, $C$. nobile, which is involved in the sesquiterpenoids biosynthesis. Multiple sequence alignment showed that CnPMK had a high similarity with other plant PMK genes. The phylogenetic tree analysis also showed that the CnPMK might keep a strong conservation during the molecular evolution. The cloning and characterization of CnPMK will provide a theoretical basis for increasing the content of sesquiterpenoids in $C$. nobile through genetic engineering. 


\section{Conflict of interest statement}

Authors declare that they have no conflict of interest.

\section{Acknowledgement}

This work was supported by the National Natural Science Foundation of China (31400603), International Science and Technology Cooperation Project of Hubei Province (2013BHE039 and 2013BHE039).

\section{References}

Bohlmann, J., Keeling, C. I., 2008. Terpenoid biomaterials. Plant J. Cell Mol. Biol. 54(4), 656-669.

Chen, F., Tholl, D., Bohlmann, J., Pichersky, E., 2011. The family of terpene synthases in plants: A mid-size family of genes for specialized metabolism that is highly diversified throughout the kingdom. Plant J. 66(1), 212-229.

Cheng, A. X., Lou, Y. G., Mao, Y. B., Lu, S., Wang, L. J., Chen, X. Y., 2007. Plant terpenoids: biosynthesis and ecological functions. J. Integr. Plant Biol. 49(2), 179-186.

Guimarães, R., Barros, L., Dueñas, M., Calhelha, R. C., Carvalho, A. M., Santos-Buelga, C., 2013. Nutrients, phytochemicals and bioactivity of wild roman chamomile: A comparison between the herb and its preparations. Food Chem. 136(2), 718-725.

Hadaruga, N. G.., Hadaruga, D. I., Tatu, C., Gruia, A., Costescu, C., Lupea, A. X., 2009. Multivariate analysis (PCA) in Compositae biocompounds class. J. Agroaliment. Process. Technol. 15, 201-210.

Houten, S. M., Waterham, H. R., 2001. Nonorthologous gene displacement of phosphomevalonate kinase. Mol. Genet.
Metabol. 72(3), 273-276.

Ma, C. M., Winsor, L., Daneshtalab, M., 2007. Quantification of spiroether isomers and herniarin of different parts of matricaria matricarioides, and flowers of Chamaemelum nobile. Phytochem. Anal. 18(1), 42-49.

Ma, S. M., Garcia, D. E., Redding-Johanson, A. M., Friedland, G. D., Chan, R., Batth, T. S., 2011. Optimization of a heterologous mevalonate pathway through the use of variant hmg-coa reductases. Metabol. Eng. 13(5), 588-597.

Miziorko, H. M., 2011. Enzymes of the mevalonate pathway of isoprenoid biosynthesis. Arch. Biochem. Biophy. 505(2), 131-143.

Rajabi Memari, H., Pazouki, L., Niinemets, Ü., 2013. The biochemistry and molecular biology of volatile messengers in trees. In: Biology, Controls and Models of Tree Volatile Organic Compound Emissions. Springer Netherlands. pp.47-93.

Redding-Johanson, A. M., Batth, T. S., Chan, R., Krupa, R., Szmidt, H. L., Adams, P. D., 2012. Targeted proteomics for metabolic pathway optimization: application to terpene production. Methods Mol. Biol. 944(2), 237-249.

Singh, P., Batth, T. S., Juminaga, D., Dahl, R. H., Keasling, J. D., Adams, P. D., 2012. Application of targeted proteomics to metabolically engineered Escherichia coli. Proteomics. 12(8), 1289-1299.

Smit, A., Mushegian, A., 2000. Biosynthesis of isoprenoids via mevalonate in archaea: the lost pathway. Genome Res. 10(10), 1468-1484.

Srivastava, J. K., Shankar, E., Gupta, S., 2010. Chamomile: a herbal medicine of the past with bright future. Mol. Med. Rep. 3(6), 895-901.

Vranová, E., Coman, D., Gruissem, W., 2013. Network analysis of the mva and mep pathways for isoprenoid synthesis. Annu. Rev. Plant Biol. 64(1), 463-476.

\section{How to cite this article:}

Yan, J., Meng, X., Xu, F., Chang, J., 2016. Molecular cloning and sequence analysis of a phosphomevalonate kinase gene (CnPMK) from Chamaemelum nobile. Int. J. Curr. Res. Biosci. Plant Biol. 3(10), 157-162. doi: http://dx.doi.org/10.20546/ijcrbp.2016.310.019 